\title{
Cardiac magnetic resonance in the assessment of hypertrophic cardiomyopathy phenotypes and stages - pictorial review
}

\author{
Magdalena Stachera ${ }^{1, A, B, C, D, E, F}$, Paweł Przybyło ${ }^{2, B}$, Katarzyna Sznajder ${ }^{1, A}$, Marek Gierlotka ${ }^{3, A, C, D}$ \\ 'Clinical Department of Diagnostic Imaging, University Hospital, Institute of Medical Sciences, University of Opole, Poland \\ ${ }^{2}$ Department of Cardiology, University Hospital in Opole, Poland \\ ${ }^{3}$ Department of Cardiology, University Hospital, Institute of Medical Sciences, University of Opole, Poland
}

\begin{abstract}
The aim of this paper is to present recent advances in hypertrophic cardiomyopathy (HCM) diagnosis and treatment based on a literature review. Special emphasis has been placed on the role of cardiac magnetic resonance imaging (CMR) for the assessment of morphological and functional consequences of different stages of HCM including prognostication. The text is illustrated with the images and data of the HCM patients diagnosed with CMR study in our hospital. CMR is an important tool, particularly relevant in novel risk factors and LV dysfunction groups. The HCM group with overt left ventricular dysfunction is underrecognized, often labelled by clinicians as dilated cardiomyopathy. Advanced diagnostic and management strategies effectively influence the natural history of HCM.
\end{abstract}

Key words: magnetic resonance imaging, cardiomyopathy, hypertrophic, phenotype.

\section{Introduction}

In this paper we refer to the phenotypes and stages described by Olivotto et al. [1], adapted by Soler et al. [2] and Muresan et al. [3].

\section{Diagnosis and prevalence}

The guidelines on diagnosis and management of hypertrophic cardiomyopathy have been issued by European Society of Cardiology (ESC) in 2014 [4] and by American College of Cardiology Foundation and American Heart Association (ACCF/AHA) in 2011 [5]. There are certain differences in disease definition in the above-mentioned documents.

On the one hand, according to ESC, in an adult, hypertrophic cardiomyopathy (HCM) is defined by a wall thickness $\geq 15 \mathrm{~mm}$ in one or more left ventricular (LV) myocardial segments - as measured by any imaging technique (echocar- diography, cardiac magnetic resonance imaging [CMR] or computed tomography $[\mathrm{CT}]$ ) - that is not explained solely by loading conditions. The clinical diagnosis of HCM in first-degree relatives of patients with unequivocal disease (left ventricular hypertrophy $[\mathrm{LVH}] \geq 15 \mathrm{~mm}$ ) is based on the presence of otherwise unexplained increased LV wall thickness $\geq 13 \mathrm{~mm}$ [4]. This definition aligns with everyday clinical practice. In this concept, hypertrophic cardiomyopathy is an 'umbrella' term that encompasses a diverse and complex spectrum of genetic and acquired diseases [4].

On the other hand, ACCF/AHA defines HCM as unexplained LV hypertrophy associated with nondilated ventricular chambers in the absence of another cardiac or systemic disease that itself would be capable of producing the magnitude of hypertrophy evident in a given patient [5]. This definition is not compatible with advanced-stage disease and multi-system disorders.

In up to $60 \%$ of adolescents and adults with HCM, the disease is an autosomal dominant trait caused by muta-

Correspondence address:

Magdalena Stachera, Clinical Department of Diagnostic Imaging, University Hospital, Institute of Medical Sciences, University of Opole, Poland, e-mail: magstachera@gmail.com

Authors' contribution:

A Study design - B Data collection - C Statistical analysis · D Data interpretation - E Manuscript preparation · F Literature search · G Funds collection 
tions in cardiac sarcomere protein genes. Mutations in the genes encoding beta-myosin heavy chain ( $M Y H 7)$ and myosin-binding protein $\mathrm{C}(M Y B P C 3)$ account for most cases $[4,6]$. The most common mode of inheritance is autosomal dominant $[4,6]$.

HCM is the most common heritable cardiomyopathy [6], traditionally believed to affect $\sim 1$ in 500 people. Recent investigations suggest even greater prevalence - a total of $1.4 \%$ participants had unexplained left ventricular hypertrophy in a population-based CMR study [7]. Lately, the prevalence of HCM has been estimated at $0.16 \%$ to $0.29 \%$ ( $\approx 1: 625-1: 344$ individuals) in the general adult population [8]. It is likely that HCM affects approximately 20 million people globally [9]. That means most persons do not receive a diagnosis during their lifetime [9]. The underrecognition of HCM has disproportionately affected women [9]. However, there is no clear connection between the specific mutation and the HCM phenotype [8], and there is no specific role of genotyping in risk stratification [9]. Crucial management decisions in patients with HCM are based on clinical and imaging criteria [9].

Expression of the HCM phenotype is variable; penetrance is incomplete and age-dependent [7]. Hypertrophy becomes visible in approximately half of genotypepositive patients by the third decade of life, and approximately three-fourths become phenotype-positive by the sixth decade [8].

Another interesting feature of sarcomere protein mutations is pleiotropy. Mutations in the same gene could manifest as HCM, dilated cardiomyopathy (DCM), restrictive cardiomyopathy, and left ventricular non-compaction (LVNC) cardiomyopathy $[1,8]$.

Genetic testing can also help to identify metabolic and storage phenocopies [8,9], present in about $10-15 \%$ of the patients [4].

Recent advances in noninvasive imaging - mostly scintigraphy utilizing bone tracers, T1-mapping CMR, and genetic testing have permitted a much better recognition of cardiac amyloidosis [10-12]. Some of the patients were initially misdiagnosed as HCM $[11,12]$. Among patients referred with initial diagnosis of HCM, the most common phenocopy is cardiac amyloidosis (9\% prevalence); Anderson-Fabry (AF) disease was diagnosed only in $2 \%$ $[11,12]$. Differential diagnosis is of particular importance in early recognition, following the development of specific disease-modifying treatments: tafamidis in transthyretinmediated cardiac amyloidosis, enzyme therapy for AF disease $[11,13]$ (Figure 1).

\section{The role of cardiac magnetic resonance imaging}

CMR is now considered an indispensable, complementary tool in the diagnosis and prognostication of HCM $[3,7,14,15]$. Most patients are initially imaged by echocardiography [3-5], but not all myocardial segments may be adequately visualized $[3,7,15,16]$. Clinically relevant discrepancies in LV maximal thickness measurements are common between the 2 techniques, and CMR is much more accurate $[7,14-16]$; we observed this also in our group of patients. The fact is of clinical importance because maximal LV wall thickness is not only the diagnostic criterion, but it is also an important risk factor [4,17]. Moreover, only basal septal and posterior wall thickness are measured routinely and documented in a standard echocardiography record. CMR can identify hypertrophy of anterior, lateral wall, and apex undetected by echocardiography, because it covers the entire ventricle with high resolution and contrast with excellent endocardial visibility [7,15-17]. Practical importance is illustrated in [18], where CMR recognized 20 more cases of HCM missed by echocardiography in a population of 155 athletes with abnormal ECG.

CMR enables quantitative assessment of LV mass, hypertrophy of the RV wall, elongated mitral valve leaflets
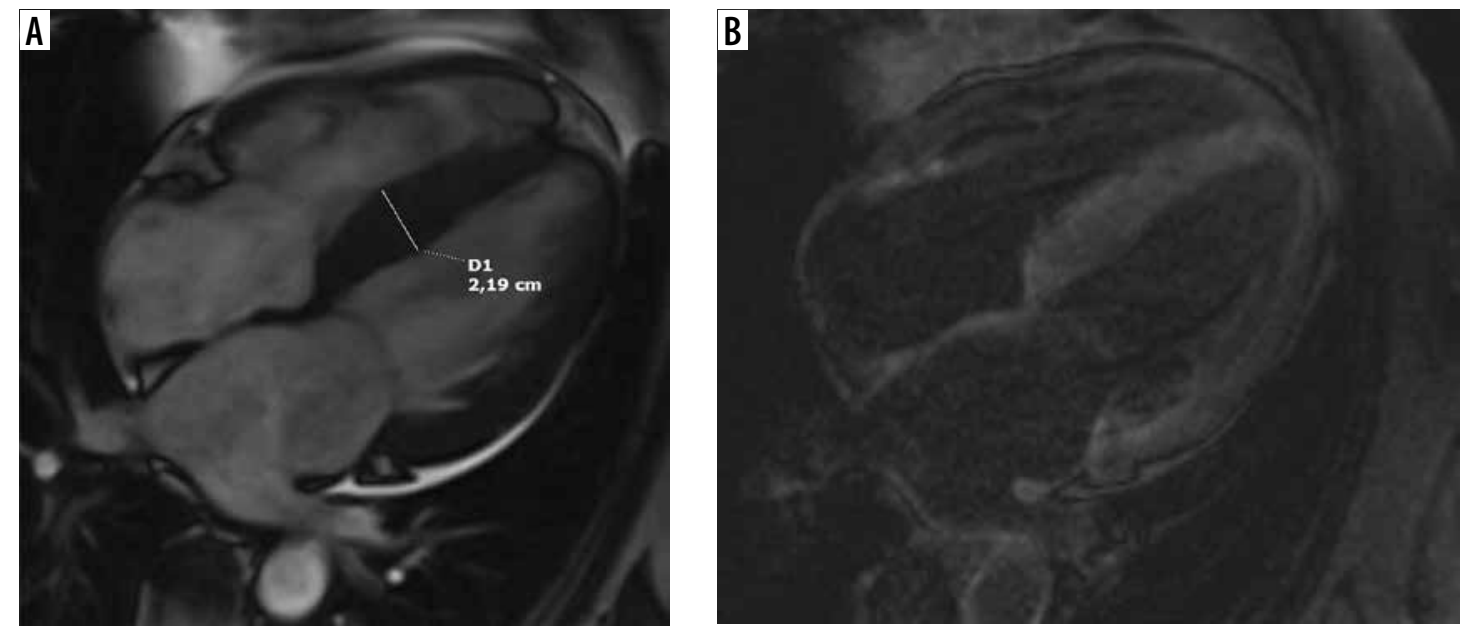

Figure 1. A 67-year-old woman with paroxysmal atrial fibrillation and hypertrophic cardiomyopathy diagnosed by echocardiography presented with congestive heart failure, EF - 30\%. Her coronary angiogram was normal. (ardiac magnetic resonance 4-chamber view: A) cine image demonstrating interventricular septum thickening, B) LGE image with suppressed blood pool and transmural enhancement of myocardium typical for amyloidosis; endomyocardial biopsy revealed SSA, genetic testing confirmed hereditary TTR amyloid; recurrent hospitalisations due to advanced heart failure during follow-up 
contributing to LVOTO, and late onset of hypertrophy in adults $[14,16,17]$. It is postulated that the diagnostic criteria for HCM in CMR may require refinement because of differences in normal wall thickness by segment, sex, and body size [7]. In [19] normal values of left ventricular myocardial thickness for every segment are given. A unique feature of CMR is tissue characterization. LGE provides accurate, non-invasive assessment of regional myocardial fibrosis with histological validation, while diffuse interstitial myocardial fibrosis is quantified by postcontrast T1 mapping - ECV [2,13,20-22]. The examples of LGE patterns in HCM patients are shown in Figure 2.

Chan et al. [23] showed that \%LGE $\geq 15 \%$ of total LV mass was associated with a 2 -fold increase in SCD at 5 years compared to patients with no LGE. Conversely, the absence of LGE was associated with lower risk of SCD [23]. The findings were confirmed by meta-analysis in 2016 [24] and could not be incorporated in previously published guidelines [4,5]. Extensive LGE was also predictive of adverse LV remodelling and progression to systolic dysfunction (end-stage HCM) [23]. In a recent study of 2094 patients with HCM (17 years of experience) a novel risk factor for SCD was proposed to enhance the American College of Cardiology/American Heart Association Strategy for Prevention of Sudden Cardiac Death in HighRisk Patients With Hypertrophic Cardiomyopathy - "late gadolinium enhancement - identified fibrosis with diffuse and extensive distribution, either quantified (usually comprising about $15 \%$ or more of LV mass) or estimated by visual inspection to be extensive and diffuse, either alone or associated with other markers" [25]. Other novel risk factors proposed in [25] are end-stage disease and LV apical aneurysm with associated contiguous regional scarring.

Global ECV was superior to all tested clinical and CMR parameters including LGE and native T1 mapping to identify HCM patients with increased risk for SCD in the recent study of $73 \mathrm{HCM}$ patients [26].

Strain analysis is a new promising tool; however, the type of algorithm employed during strain assessment has a significant impact on the results [27].

Our illustrative material consists of 91 studies of 88 patients referred for CMR at the Department of Diagnostic Imaging of the University of Opole Hospital from 2011 to 2019. The images were retrospectively analysed with dedicated software: syngo.via MR Cardiac Analysis (Siemens Healthineers), including LGE quantification, volume/time curve parameters, and LA volumetry.

We quantitated LGE in all our patients as LGE volume and \% LGE mass. An alternative method of simplified, quick estimation of LGE in HCM suggested by Kłopotowski et al. in [28] was also used. Unfortunately, we do not have the possibility of myocardial mapping in our CMR laboratory yet; we would not be able to apply this measurement retrospectively anyway.

Additionally, the volume/time curve parameters were derived from cine images used for functional parameter estimation, as suggested in [29,30]. The software provided in syngo.via MR Cardiac Analysis calculates PER, PET, PFR, and PFT. The practical application of such data is difficult [31] because of inconsistent values of normal ranges provided by different authors [19,29,32], probably due to software differences.
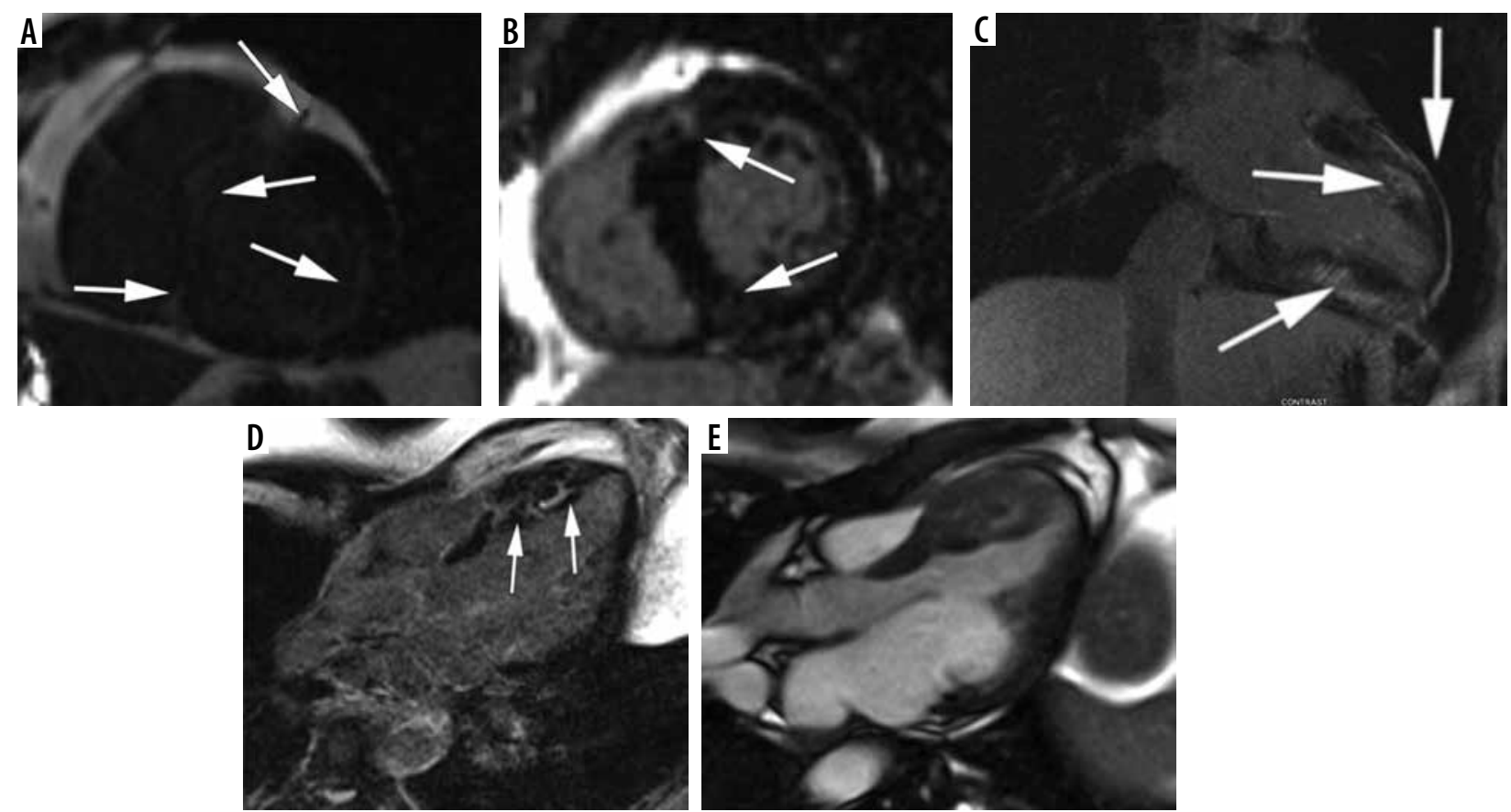

Figure 2. Most common LGE distribution patterns in hypertrophic cardiomyopathy. A) Extensive midwall LGE in short-axis view (arrows). B, C) Patchy enhancement at insertion points of the RV wall into the anterior and posterior interventricular septum (arrows): B) short-axis view, C) modified 2-chamber view, D) patchy mid-wall delayed enhancement in the hypertrophied segments (arrows), E) cine image corresponding with D 
The other parameters we assessed were left atrium volumetric, including LAEDV, LAVI, and LAEF acquired by biplane area method, as in [33-35]. In LAEF $(<38 \%)$, LAEDV ( $>118 \mathrm{ml}$ ) and age ( $\geq 40$ years) identified HCM patients at risk for development of AF [34].

\section{Stage l: Non-hypertrophic HCM}

Due to genetic testing, a new HCM subgroup has been identified: HCM - causing mutation carriers without LV hypertrophy, known as "genotype-positive-phenotypenegative" [36]. With CMR imaging; however, it is possible to discover a certain degree of cardiac hypertrophy [1], and other abnormalities like myocardial crypts, elongated mitral valve leaflets, accessory papillary muscles, and fibrosis $[2,3,22]$ - see Figure 3. ECG abnormalities may be evident [1].

We had no patients in this group, because no program of systematic genetic screening was conducted. One of our patients, however, a 40-year-old woman with a family history of HCM presented with chest pain, dyspnoea, reduced exercise tolerance, palpitations, and anaemia. CMR revealed an abnormal papillary muscle and borderline hypertrophy of $13 \mathrm{~mm}$ (Figure 3).

In [37] it was postulated that the identification of elongated mitral leaflets by CMR can represent a clinical marker in HCM family members without hypertrophy, whose genotype is unknown. In [35] Fahrad et al. demonstrated that LA dysfunction measured as decreased LAEF is detectable by CMR in preclinical HCM mutation carriers.
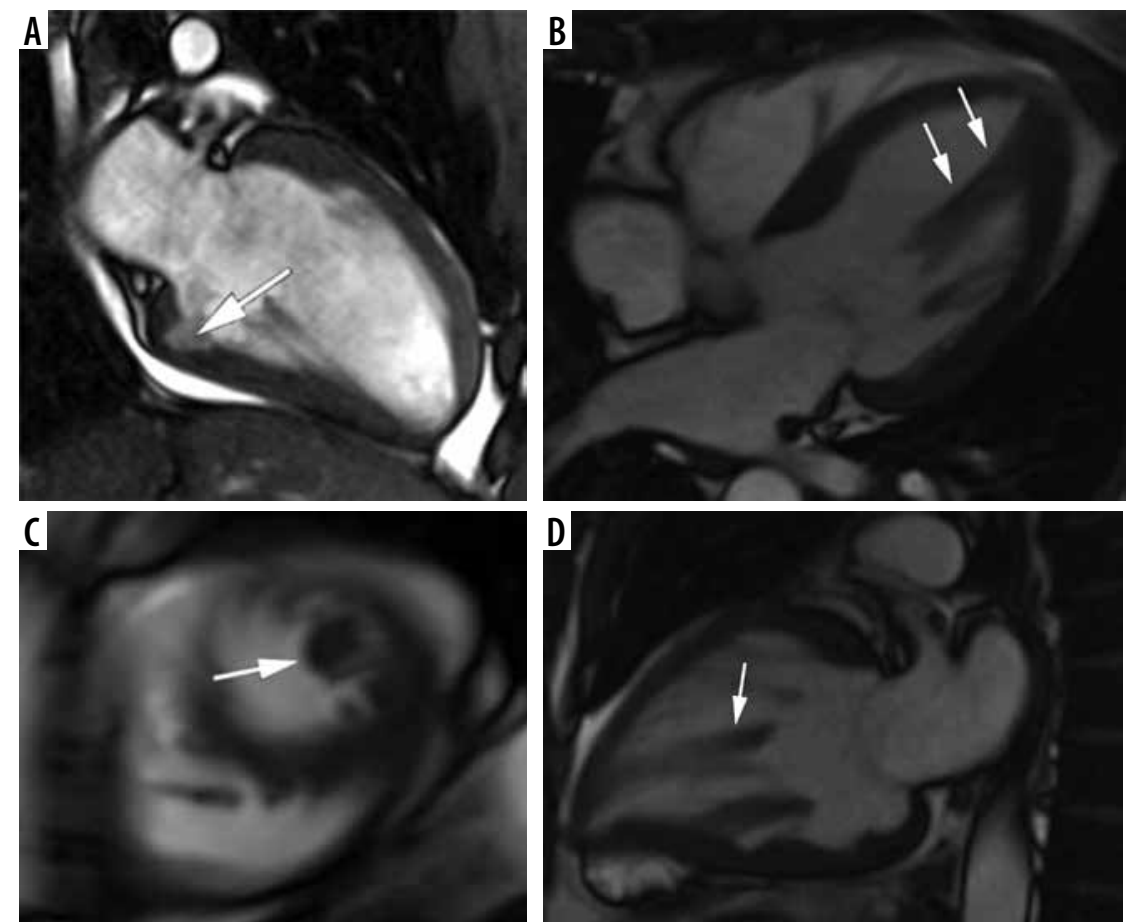

Figure 3. SSFP cine images - examples of findings characteristic for hypertrophic cardiomyopathy (HCM) - causing mutation carriers. A) Cardiac magnetic resonance imaging (CMR) image at end - diastole, 2-chamber view, showing myocardial crypt perpendicular to the endocardial border at inferior wall (arrow). B, C, D) 40-year-old woman with a family history of HCM presented with chest pain, dyspnoea, reduced exercise tolerance, palpitations, and anaemia. CMR SSFP cine images revealed abnormal papillary muscle (arrows) and a mild hypertrophy; B) 4-chamber view, C) short-axis view, D) 2-chamber view 

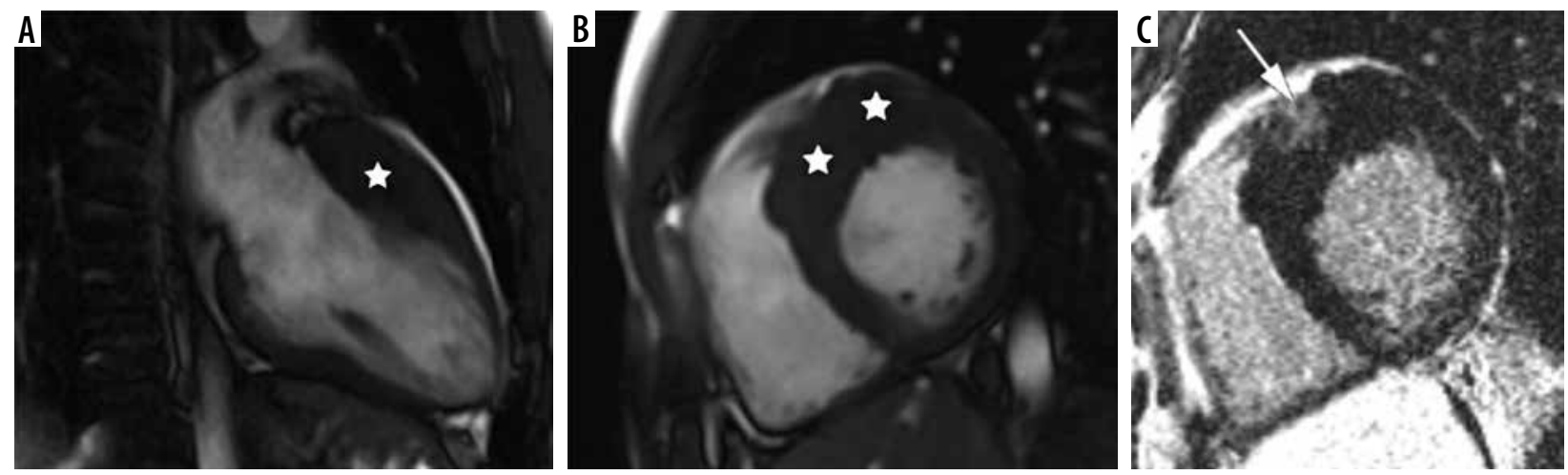

Figure 4. Distribution of hypertrophy - basal anteroseptal. A 29-year-old woman who presented with palpitations. A) Two-chamber SSFP cine cardiac magnetic resonance imaging image at end-diastole shows anterior hypertrophy (asterisk). B) Short-axis cine view demonstrates marked anteroseptal hypertrophy (asterisks). C) Corresponding LGE image showing the area of delayed enhancement (arrow)
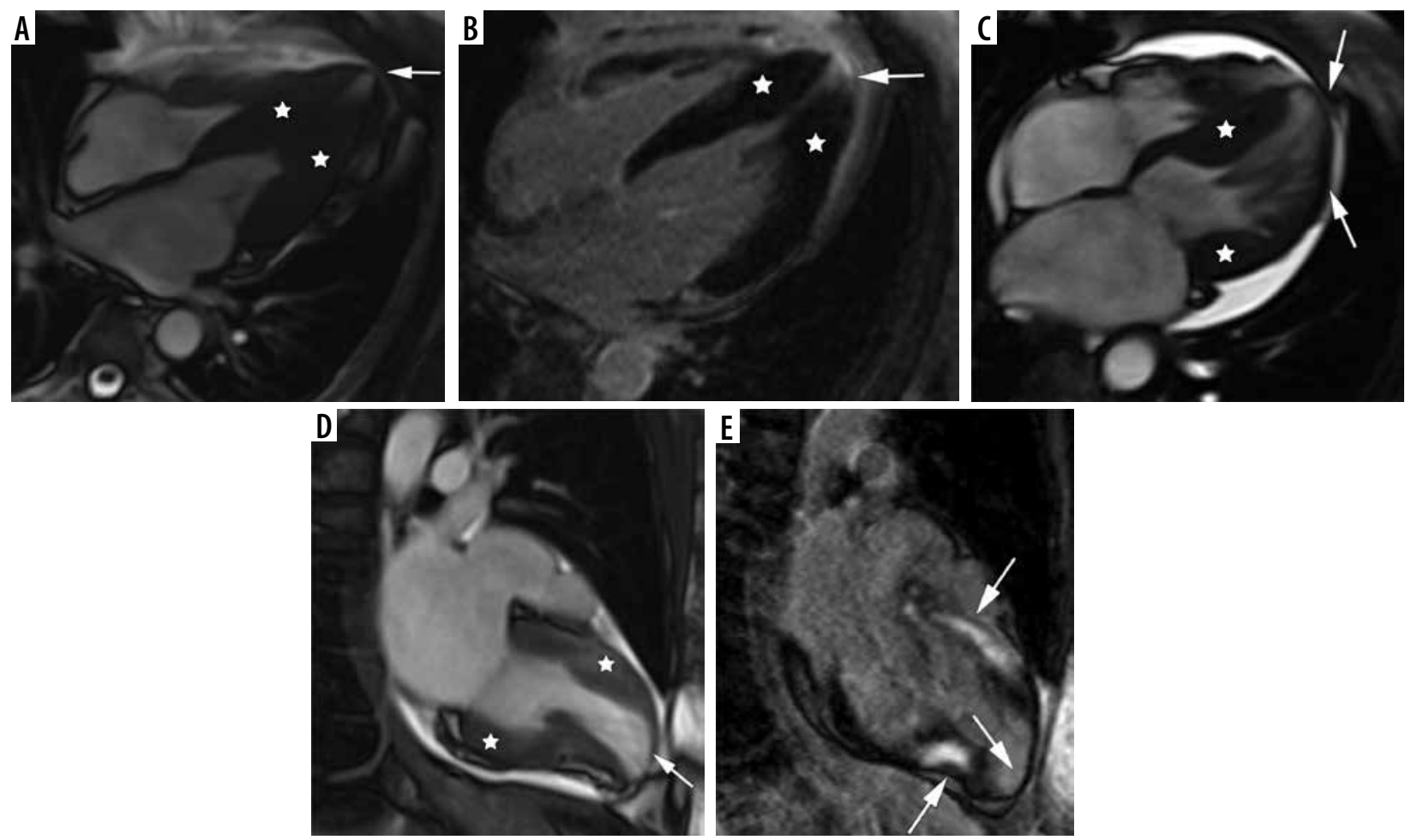

Figure 5. Distribution of hypertrophy - reversed curvature with apical aneurysm. A 53-year-old man referred for cardiac magnetic resonance imaging (CMR) with a suspicion of hypertrophic cardiomyopathy (HCM), EF - 75\%. A) Systolic 4-chamber SSFP cine image demonstrates a "dumbbell" configuration of the LV cavity, mid-cavity constriction by hypertrophied segments (asterisks) and a thin-walled apical aneurysm (arrow), not visible in echo. B) LGE image in diastole showing hypertrophy (asterisks) and a subtle delayed enhancement thinned apical aneurysm (arrow). A 57-year-old woman with recurrent syncope and nSVT, suspected hypertrophic cardiomyopathy: C) systolic cine 4-chamber view, demonstrating hypertrophy (asterisks), thin-walled apical aneurysm (arrow), and relatively thin lateral wall (arrow), D) systolic cine 2-chamber view, demonstrating hypertrophy (asterisks) and thin-walled apical aneurysm (arrow), E) LGE 2-chamber view showing mid-wall, patchy delayed enhancement of the hypertrophied segments (thick arrows) and no enhancement of the aneurysm (thin arrow); EF was in the adverse remodelling range - 58\%. The aneurysm was not visible in the echo examination. The patient refused ICD and was hospitalised because of arrhythmia and heart failure during the follow-up

tion can be considered in these patients [40]. In some series $\mathrm{LV}$ apical aneurysms are associated with a $10 \%$ annual event rate [40], but the ESC guidelines do not recommend ICD placement based only on this risk factor [4].

In our group only 6 patients presented with midventricular hypertrophy in classic phenotype $(9.6 \%)$. Three (3.4\% of all HCM patients) developed small $(<2 \mathrm{~cm})$ apical aneurysm without thrombi, 1 in classic phenotype, and 2 in stage III adverse remodelling. None of them was discov- ered by echocardiography. Contrary to [40], only 1 of our patients exhibited LGE in the aneurysm.

An important variant of hypertrophy is apical distribution, usually present in 5-25\% of patients [2] - see Figure 6. In our series apical hypertrophy was present in 10 people - $16 \%$ of classic phenotype.

Spade-like configuration of the LV cavity and giant inverted anterolateral $\mathrm{T}$ waves on the electrocardiogram are usually present $[2,3,21,22]$. Cardiac MRI is strongly 

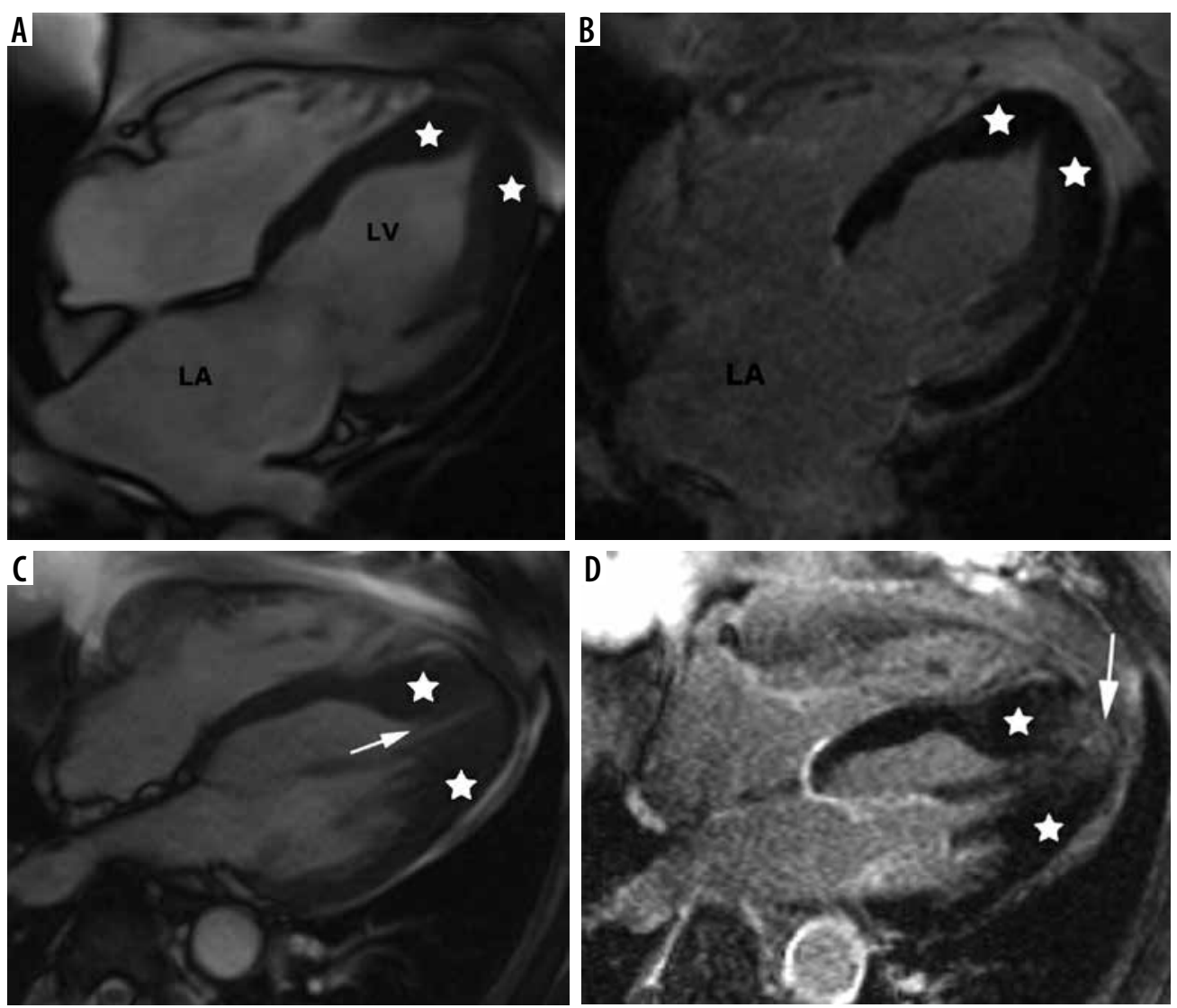

Figure 6. Distribution of hypertrophy - apical involvement, 4-chamber views. A, B) A 61-year-old woman previously diagnosed with hypertrophic cardiomyopathy (HCM) presented with palpitations - paroxysmal AF and nsVT, EF - 64\%; cardiac magnetic resonance imaging (CMR) revealed mild hypertrophy of the apex (asterisks), please note large left atrium and B) no LGE; the patient received ICD and underwent two cryoablations because of recurrent $A F$ and was hospitalised because of heart failure due to arrhythmia during the follow-up. C, D) A 58-year-old man referred for CMR because of suspected HCM in echo, apical hypertrophy more pronounced than in A, B. C) Cine image demonstrate spade-like appearance of LV (triangle). D) LGE image showing enhancement in hypertrophied apical segments; uneventful follow-up

recommended to diagnose and evaluate apical HCM because echocardiography has significant limitations in this region, in particular in correct differentiation of apical hypertrophy from LV noncompaction [21,22].

In a certain subgroup of patients hypertrophy also involves the RV wall - see Figure 7.

We did not observe concentric, symmetric hypertrophy in classic phenotype - as in [2], contrary to [3,22].

Recently the RESTYLE - HCM trial has shown a significant antiarrhythmic effect of the late sodium current inhibitor ranolazine on ventricular ectopic burden in nonobstructive HCM. The drug can also be used to control anginal symptoms; however, the trial does not support the use to improve functional capacity and diastolic dysfunction.

\section{Left ventricular tract obstruction}

Patients with left ventricular tract obstruction (LVOTO) constitute an important subgroup of the classic HCM phenotype - hypertrophic obstructive cardiomyopathy (HOCM).

According to the literature, up to $70 \%$ of patients with classic HCM may have resting or provocable LVOTO $[2,37]$. Exercise (stress) echocardiography is currently considered the method of choice to provoke LVOT gradients, when absent at rest [9]. The resting gradient can also be quantified by phase contrast CMR sequences, although it increases the scanning time and may underestimate the values $[2,3,21]$. Gradient $>30 \mathrm{mmHg}$ is considered significant, and in the presence of severe, drug-refractory symptoms $>50 \mathrm{mmHg}$ may be considered an indication for invasive septal reduction therapies (SRT) including surgical myectomy (SM) or alcohol septal ablation (ASA) [41]. New pharmacological approaches e.g. a novel myosin inhibitor (mavacamten) - may change therapeutic options in HOCM patients [41].

Cine MRI in a long-axis view can provide an accurate picture of the precise mechanism of outflow tract obstruction [2], see Figure 8. Turbulent flow is visibly generated by SAM and movement of subvalvular apparatus toward the basal interventricular septum, producing a posteriorly directed mitral regurgitant jet [2]. Elongated mitral leaflets associated with small LVOT represent an important contribution to LVOTO, and the ratio of AML/LVOT with a cut-off value of 2 was evaluated in [37], also in the context of most appropriate septal reduction strategy. Surgical myectomy can be combined with mitral valve correction, whereas ASA cannot. 

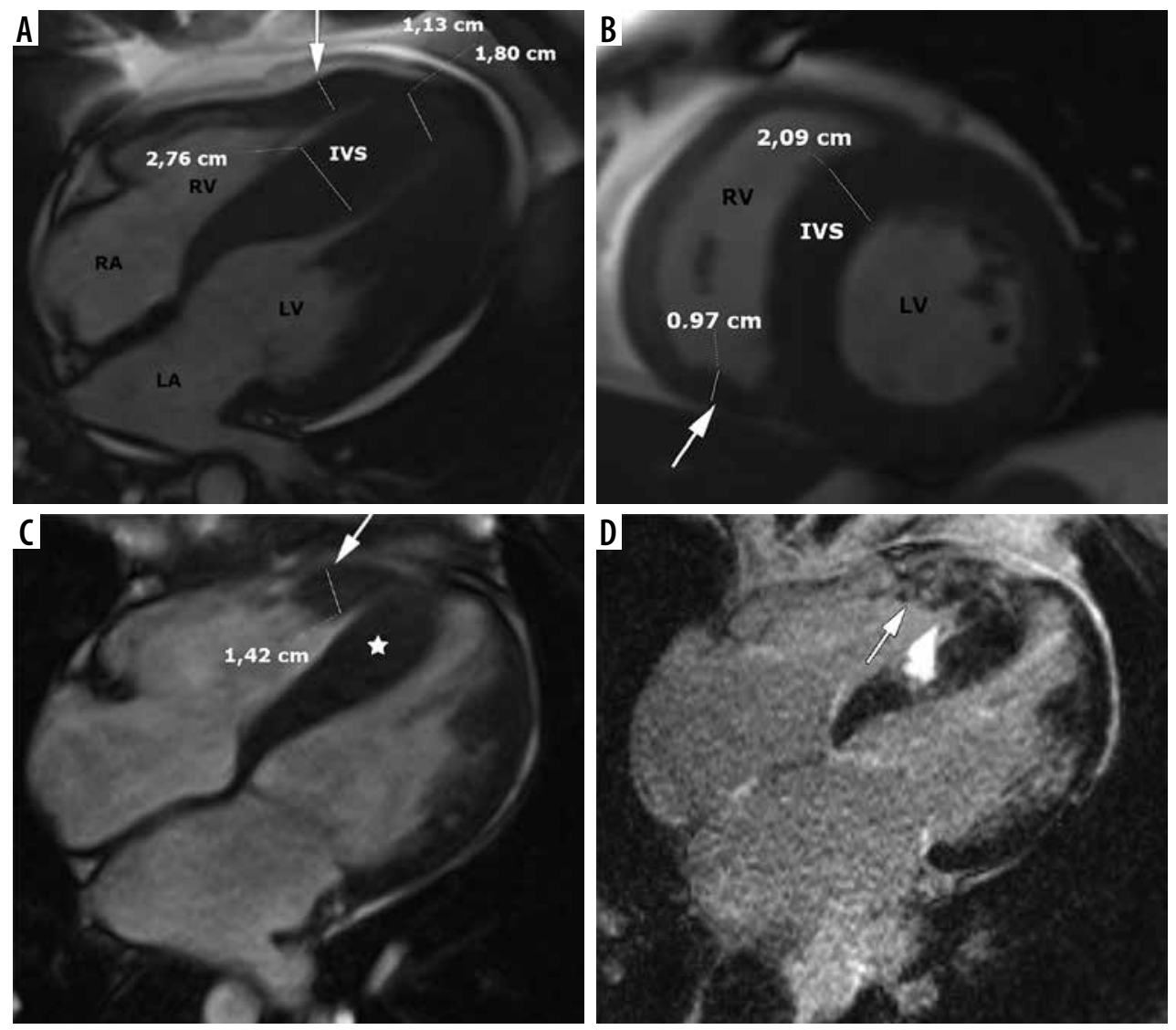

Figure 7. Distribution of hypertrophy with RV involvement. A, B) A 39-year-old gentleman with massive biventricular hypertrophy shown in SSFP cine images, arrow indicates free right ventricular wall, EF - 73\%.C, D) A 36-year-old gentleman, referred for routine cardiac magnetic resonance imaging after alcohol septal ablation, EF-59\%. C) SSFP cine end-diastolic four-chamber image demonstrates IVS (asterisk) and free RV wall (arrow) hypertrophy. D) 4-chamber LGE image demonstrates patchy areas of delayed enhancement present also in hypertrophied RV wall (arrow) and probably post-ablation septal scar
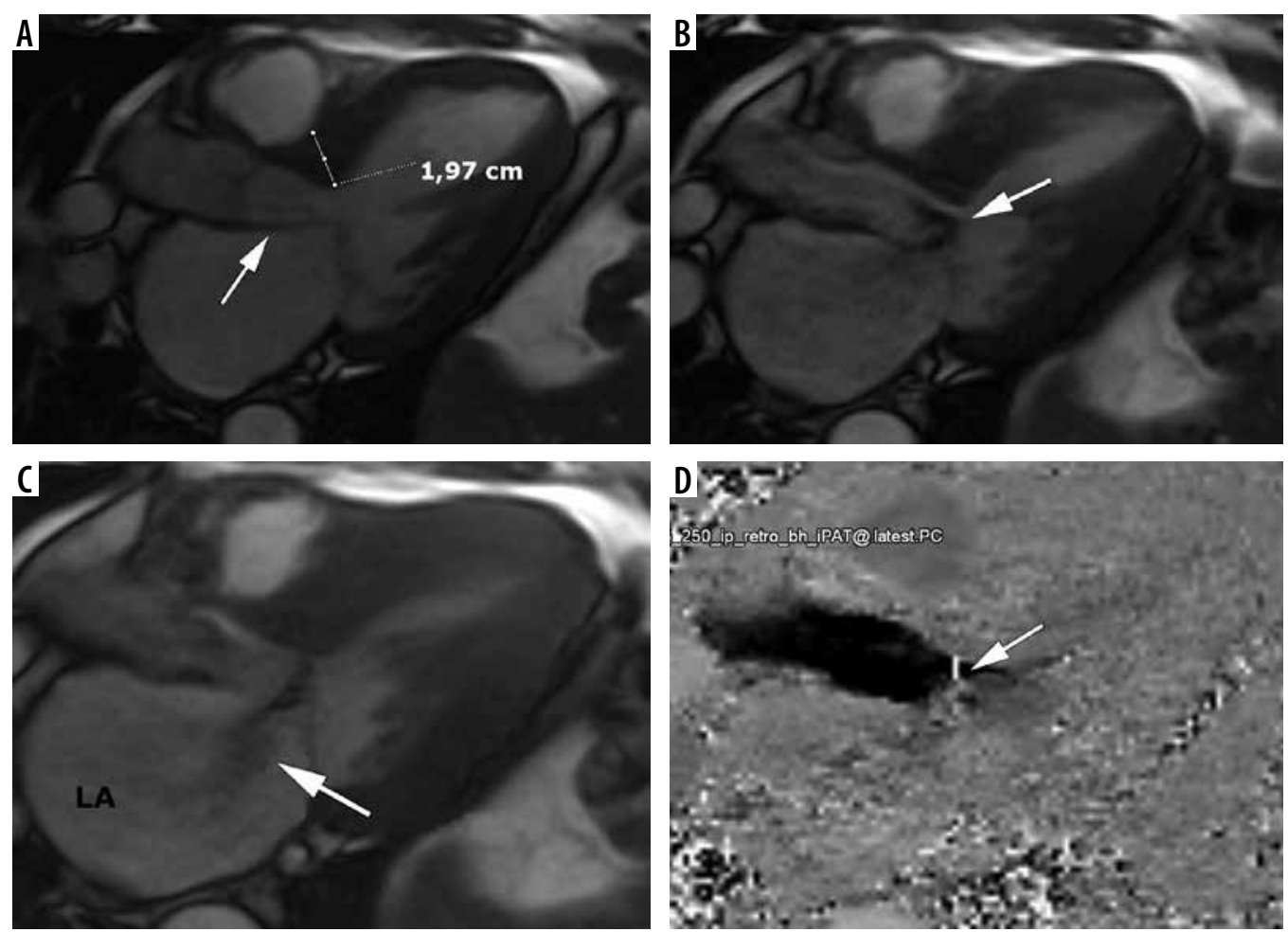

Figure 8. Left ventricular outflow tract obstruction: a 56-year-old gentleman diagnosed with obstructive hypertrophic cardiomyopathy referred for routine cardiac magnetic resonance imaging. A) Three-chamber diastolic cine image show anteroseptal hypertrophy and elongated anterior mitral leaflet (arrow), B) mid-systolic image show SAM and turbulent-velocity jet (arrow) within the LVOT, C) late-systolic image demonstrates posterior jet of dynamic mitral regurgitation caused by SAM; note enlarged left atrium, D) in-plane phase velocity image showing LVOTO high-velocity jet (arrow). Maximal LVOT velocity was $5.5 \mathrm{~m} / \mathrm{s}$, mitral regurgitation volume $-52 \mathrm{ml}$ 
Another interesting CMR parameter - the LVOT/ Ao diameter ratio differed significantly among the $3 \mathrm{sub}$ groups: non-obstructive $0.60 \pm 0.13$, latent $0.41 \pm 0.16$, obstructive $0.24 \pm 0.09$ [42]. In our cohort, LVOTO visible in the cine long-axis view was present in 20 patients - $32 \%$ of classic phenotype. The calculated pressure gradient range was between 9 and $148.8 \mathrm{mmHg}$. Most of these patients exhibited LVOT/Ao ratio $<0.5$, in all of them the ratio was $<0.54$ and AML/LVOT $>2$ with one outsider whose echocardiography demonstrated LVOTO. In all patients without visible obstruction LVOT/Ao $\geq 0.58$. AML and PML were elongated compared to the normal values listed in [37].

Pressure gradient correlated well with LVOT/Ao and AML/LVOT ratios. Higher PET and PER and high EF probably reflect the hyperdynamic state.

Two of our patients underwent SRT in the past: 1 ASA and 1 septal myectomy simultaneously with CABG.

\section{Stage III: Adverse remodelling}

Adverse remodelling is defined by progressive decreases in systolic function (EF 50-65\%) superimposed to the classic HCM phenotype $[1,2]$. This stage is characterized by concomitant reduction or loss of LVOTO, progressive LV wall thinning, increasing LV fibrosis with relatively preserved clinical and haemodynamic balance [1-3] see Figure 9. This seems to represent a selective pathway followed by about 15 to $20 \%$ of HCM patients, with the minority progressing to overt dysfunction [1]. Both LV and LA remodelling are common, and AF frequently leads to clinical progression. The long-term outcome of this group is not known, but cardiac mortality is estimated to be $3-5 \%$ per year [1]. It is striking that although this EF would be considered within normal range for other populations, in [43] it was shown that HCM patients in this group have a nearly 3 -fold increased risk of developing overt left ventricular dysfunction. It was postulated that they would probably benefit from closer management [43].

The question arises: what is the trigger for adverse LV remodelling and dysfunction? Olivotto in [1] speculates that it is internal, noting severe HCM progression in patients with complex genotypes - double or triple sarcomeric mutations. External factors such as viral myocarditis or epicardial coronary disease in his opinion are only anecdotally associated. On the other hand, Frustaci et al. in their intriguing paper [44] detected histological signs of overlapping active myocarditis and a viral genome in 28 of $42 \mathrm{LV}$ endomyocardial biopsy tissue samples from HCM patients with acute clinical deterioration. A viral genome was present in 14 of 28 patients with overlapping myocar-
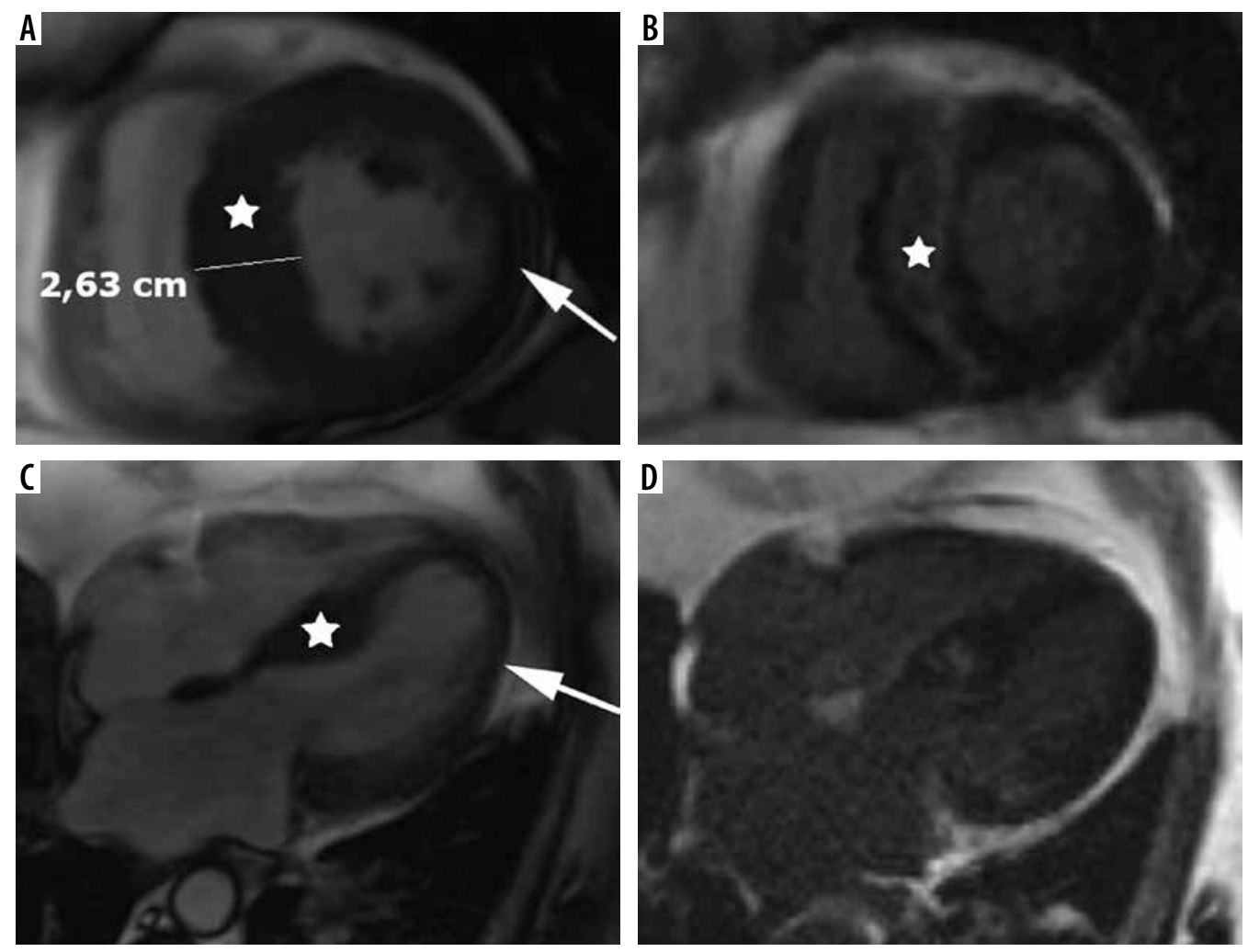

Figure 9. Adverse remodelling. A 71-year-old man admitted after cardiac arrest caused by VT/VF, with persistent AF. He was diagnosed with hypertrophic cardiomyopathy (HCM) 1 year earlier, with an EF 55-60\%, SAM, LVOT0, normal coronary arteries. Cardiac magnetic resonance images: A) short-axis SSFP cine view showing septal hypertrophy (asterisk) and lateral wall thinning (arrow), B) same level LGE image demonstrates large midwall area of delayed enhancement in hypertrophied septum (asterisk), C) SSFP cine 4-chamber view showing septal hypertrophy (asterisk) and lateral wall thinning (arrow), D) corresponding LGE image. During CMR study no LVOTO was demonstrated, EF equalled $51 \%, \mathrm{LAVI}-75 \mathrm{ml} / \mathrm{m}^{2}, \mathrm{LAEF}-1 \%, \mathrm{LGE} 12 \%$. The patient received ICD as primary prevention, during the follow up successful shocks were delivered, due to recurrent electric storms with congestive heart failure he required frequent hospitalizations 
ditis. The authors concluded that myocarditis, often viral, represents a common cause of acute clinical deterioration in HCM. Recently Maron et al. in [39] introduced the concept of acquired HCM risk factors, postulating that nongenetic, potentially modifiable mechanisms may be involved in cardiomyopathic disease, even when there is also a genetic variant that indicates risk, similar to dilated cardiomyopathy in which the phenotype can also be triggered by various nongenetic factors such as alcohol, viral infection, and toxins [39]. Disease progression was attributed to multiple causes, including microvascular dysfunction, progressive myocardial fibrosis, and cardiomyocyte energy depletion in [41]. Disease progression may be subtle for a treating clinician due to preserved EF; in [41] the mean age at progression to heart failure is estimated to be after 65 years of age.

In our group 15 patients belonged to this stage, $17 \%$ of the whole cohort. Atrial fibrillation was documented in $5(30 \%)$. Two patients had the ICD implanted and 1 successful shock was delivered. Two of the patients were not diagnosed as HCM by echo - their maximal wall thickness measured was below $15 \mathrm{~mm}$.

\section{Stage IV: Overt dysfunction}

The high-risk phenotype of HCM with LV systolic dysfunction (LVSD), defined by an $\mathrm{EF}<50 \%$, previously called "end-stage disease (ES)" or "burnt-out" develops in a minority of HCM patients, roughly $3.5-10 \%$ in described cohorts $[1,2,45,46]$.

It is important to realize that in HCM patients EF decreases from typical supranormal values, and EF slightly below the lower limits of normal subjects is substantially reduced for this population. Literally, this group encompasses both HFrEF and HFmrEF ranges, but due to unique characteristics of HCM this classification is not adequate. LVOT gradients are absent in this stage [1].

Olivotto in [1] characterized this advanced stage of the disease as spanning between the 2 extreme morphofunctional manifestations. The first was defined as the hypokinetic-dilated form (also called D-ES = ventricular dilatation end stage by Cheng in [45]) and is characterized by progressive LV wall thinning, LV dilatation, and spherical remodelling - see Figure 10. This variant may be hard to distinguish from a primary dilated cardiomyopathy, although residual hypertrophy is often retained [1]. The other variant, hypokinetic-restrictive (also called N-ES = normal ventricular size end stage), is characterized by a small and stiff LV with extreme, progressive diastolic dysfunction, marked biatrial dilatation, and AF, whereas systolic LV function is only mildly or moderately impaired - see Figure 11. These patients may present with clinical signs of low cardiac output due to restrictive filling rather than congestion [1]. Furthermore, most are not candidates for left ventricular assist devices (LVAD) because of their relatively small cavities and residual contractile function.
Interestingly, the concept of possible myocardial recovery after LV unloading was presented in [1] and deserves further investigation in HCM LVSD patients.

In 2006 Harris et al. [46] described CMR in $6 \mathrm{HCM}$ LVSD patients, and each showed large areas of LGE indicative of fibrosis, frequently transmural. Further studies revealed that the CMR features of HCM LVSD also span between the 2 extreme forms, with dominating LV or LA remodelling. LGE is more extensive in D-ES and LAVI is larger in N-ES. Both LGE and LAVI were significant predictors of poor outcomes in [45], in which CMR scans of 63 patients with ES were analysed.

Accelerated clinical deterioration was reported to occur usually over 5-6 years $[1,2]$.

An interesting paper on HCM with LV systolic dysfunction [43] has recently been issued, combining the data from 11 high-volume HCM centres making up the international SHaRe Registry (Sarcomeric Human Cardiomyopathy Registry). Nearly 7000 HCM patients were observed from 1960 through March 2019, including 553 patients with HCM-LVSD. In this population LVSD affected around $8 \%$ of patients with HCM. Seventy-five per cent of them experienced adverse events, including $35 \%$ experiencing a death equivalent to an estimated median time of 8.4 years after developing systolic dysfunction. Risk factors of poor prognosis for HCM LVSD patients were multiple pathogenic/probably pathogenic sarcomeric variants, atrial fibrillation, and left ventricular ejection fraction $<35 \%$. Genetic substrate appeared to play a role in both prognosis (multiple sarcomeric variants) and the risk for incident development of HCM-LVSD (thin filament variants). The fate of an individual patient is difficult to predict; many patients do not experience the composite outcome [43]. The patients with LVOTO were less likely to develop systolic dysfunction. LGE was more prevalent in the patients with HCM-LVSD [43].

As far as treatment is concerned, the current guidelines systematically recommend standard HF therapy: the routine measures include ACE inhibitors, angiotensin receptor blockers, beta blockers specific for heart failure, spironolactone, and loop diuretics. Oral anticoagulants are important in cases of AF or apical aneurysm. No significant improvement occurred in the recent randomized trial with trimetazidine $[41,47]$. Data on sacubitrilvalsartan are awaited in HCM [41]. Moreover, overt LV dysfunction should be considered as a potential indication for ICD (or CRT-D) placement [25], even if this factor is not included in the ESC risk score. It is important, however, to take into account that age older than 60 years is itself associated with low likelihood of SCD [25]. Tailored surgical options may be considered: mitral plasty, LVAD, and cardiac transplantation $[1,41]$. In a recent study of 118 LVSD HCM patients [48] the contemporary natural history of ES, utilizing advanced heart failure treatment strategies, was analysed. Annual mortality was $2 \%$, and survival at 10 years was $87 \%$ (95\% CI: 67, 95). However, 

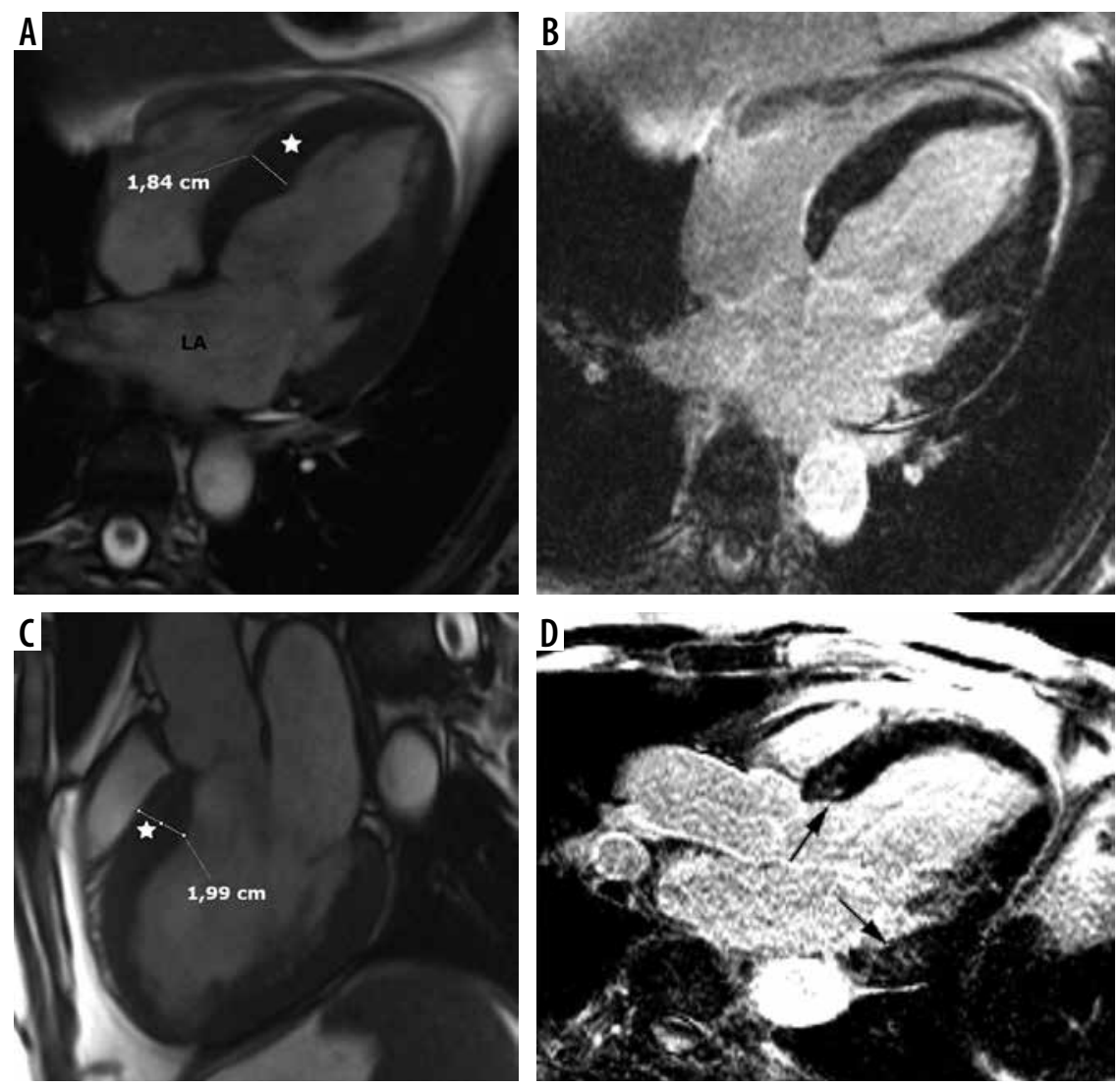

Figure 10. Overt dysfunction with hypokinetic-dilated form, D-ES. A 56-year-old man presented with decompensated CHF, low EF - 32\%, recent infection, suspected myocarditis. Cardiac magnetic resonance imaging revealed EF 24\%, LVEDV - $263 \mathrm{ml}$, LVESV - $200 \mathrm{ml}, \mathrm{LAEF}-38 \%, \mathrm{LAVI}-54 \mathrm{ml} / \mathrm{m}^{2}$, LGE 2\%. A, C) SSFP end-diastolic cine images demonstrated IVS hypertrophy inconsistent with dilated cardiomyopathy and LA enlargement. B, D) Corresponding LGE images, small, patchy areas of delayed enhancement - arrows. The patient received ICD, during follow-up 2 successful shocks were delivered, he required frequent hospitalizations due to recurrent heart failure
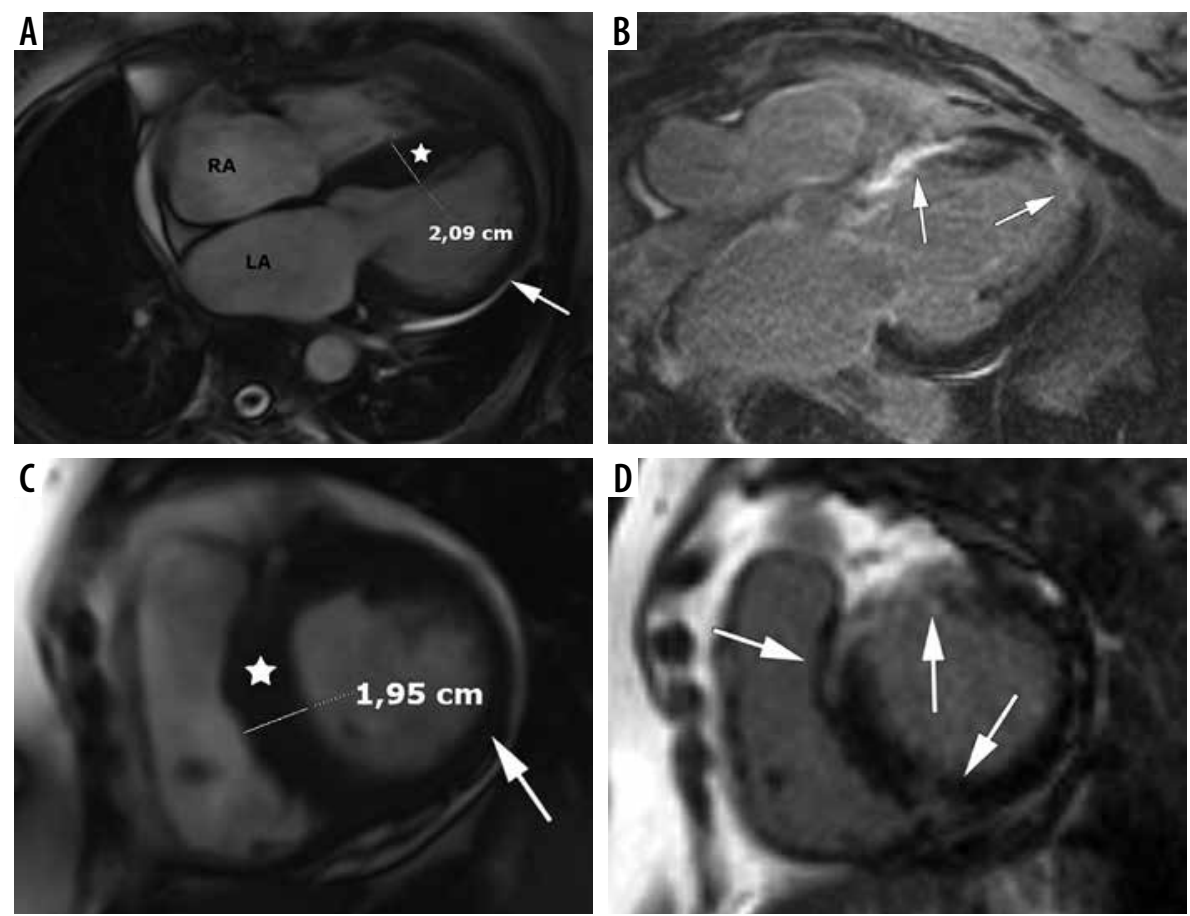

Figure 11. Overt disfunction hypokinetic-restrictive, N-ES. A 61-year-old woman with persistent AF, nsVT, with a history of ICD explantation because of infective endocarditis presented with palpitations and dyspnoea. Cardiac magnetic resonance imaging (CMR) revealed LAEF - 39\%, LAVI - $64 \mathrm{ml} / \mathrm{m}^{2}$, LVEDV - 159 $\mathrm{ml}$, LVESV - $87 \mathrm{ml}$, EF - 45\%, LGE 17\%. A) SSFP cine 4-chamber view showing septal hypertrophy (asterisk) and lateral wall thinning (arrow), note biatrial enlargement. B) corresponding LGE image showing extensive, almost transmural delayed enhancement areas (arrows). C) Cine short-axis view showing again septal hypertrophy (asterisk) and lateral wall thinning (arrow). D) Corresponding LGE image showing extensive delayed enhancement areas (arrows). Her HCM risk score was 5\%, she refused another ICD implantation and required recurrent hospitalizations because of heart failure during the follow-up 
aborted adverse HCM events (including appropriate ICD shocks, heart transplant, and resuscitated out-of-hospital cardiac arrest) were $8 \%$ per year, 4 times the HCM mortality. HCM-related deaths were 50\% lower than previously reported in ES-HCM. This highlights the potential role of appropriate intervention strategies.

Our population of patients differed substantially from previously described [1], because we did not observe a large cohort of patients for a sufficiently long period of time. In [43] systolic dysfunction developed a median of 15 years after initial diagnosis of HCM. The patients who develop LV dysfunction during follow-up are classified as incident HCM-LVSD and are lacking in our cohort. Our end-stage group consists mostly of patients referred to CMR because of heart failure symptoms and reduced EF by echo, some with initial diagnosis of myocarditis, so they are classified as prevalent HCM-LVSD [43]. Patients were designated as having HCM-LVSD in our study when CMR revealed both $\mathrm{LVMT} \geq 15 \mathrm{~mm}$ and $\mathrm{LVEF}<50 \%$. Interestingly, echocardiography revealed left ventricular maximal thickness $>15 \mathrm{~mm}$ only in 3 of them. Surprisingly, our end-stage group was dominated by men 10 out of 11 were male (90\%). It consisted of $12.5 \%$ of the whole cohort - significantly more than in [1]. No LVOTO was present. We observed 2 deaths - 1 because of progressive heart failure in a patient with ICD, and 1 extra cardiac - ruptured aortic aneurysm. Adequate ICD intervention was delivered twice in 1 patient. Three patients needed unscheduled hospitalizations. Nine suffered from concomitant hypertension. All patients presented with either LAEF $<38 \%$ or LAEDV $\geq 118 \mathrm{ml}$ - risk of AF by [34], only 1 had small atria. Only 4 of our patients had LVEDVI $\leq 90 \mathrm{ml} / \mathrm{m}^{2}$, belonging to N-ES [45]. Most of the patients in this group were described as dilated cardiomyopathy by treating physicians.

\section{Conclusions}

HCM is an extremely heterogeneous disease [1]. The patients suffering from it constantly defy rigid classifications.
The reasons for such diversity even within members of the same family are poorly understood. Epigenetic and environmental factors are postulated [1,39].

There is limited awareness among patients and physicians regarding the risk of disease progression in HCM [1], and its recognition is therefore delayed, often to the advanced and truly "end-stage" phases. The HCM group with overt left ventricular dysfunction is underrecognized, often labelled as dilated cardiomyopathy. This poses significant difficulties in assessing risk ratios and optimal therapy choices. There is a clear need to better understand HCM-LVSD to improve risk stratification and to inform clinical management.

The role of CMR is crucial, particularly in precise diagnosis, novel risk factors, and the LV dysfunction group $[15,25,45]$.

Historically, HCM was considered a rare disease with an ominous prognosis. Now, it is recognized worldwide as a relatively common genetic heart disease. Important diagnostic and management strategies effectively influence the natural history of HCM $[48,49]$.

These include CMR, stress echocardiography, refined algorithms for risk stratification, ICD, septal reduction therapies, anticoagulant therapy in patients with atrial fibrillation and LV aneurysms, and heart transplantation [9]. In specialized referral centres in the USA a recent analysis revealed that most deaths in affected patients are unrelated to HCM, with noncardiac coexisting cardiac conditions and cancer greatly influencing survival, particularly in older patients $[9,48,49]$.

\section{Conflicts of interest}

The authors report no conflict of interest.

\section{References}

1. Olivotto I, Cecchi F, Poggesi C, Yacoub MH. Patterns of disease progression in hypertrophic cardiomyopathy. Circ Heart Fail 2012; 5: 535-546.

2. Soler R, Méndez C, Rodríguez E, et al. Phenotypes of hypertrophic cardiomyopathy. An illustrative review of MRI findings. Insights Imaging 2018; 9: 1007-1020.

3. Muresan ID, Agoston-Coldea L. Phenotypes of hypertrophic cardiomyopathy: genetics, clinics, and modular imaging. Heart Fail Rev 2021; 26: 1023-1036.

4. Elliott PM, Anastasakis A, Borger MA, et al. 2014 ESC guidelines on diagnosis and management of hypertrophic cardiomyopathy: the task force for the diagnosis and management of hypertrophic cardiomyopathy of the European Society of Cardiology (ESC). Eur Heart J 2014; 35: 2733-2779.

5. Gersh BJ, Maron BJ, Bonow RO, et al. 2011 ACCF/AHA guideline for the diagnosis and treatment of hypertrophic cardiomyopathy: a report of the American College of Cardiology Foundation/ American Heart Association Task Force on Practice Guidelines. Circulation 2011; 124: e783-831. DOI: 10.1161/CIR.0b013e318223 e2bd.

6. Geske JB, Ommen SR, Gersh BJ. Hypertrophic cardiomyopathy: clinical update. JACC Heart Fail 2018; 6: 364-375.

7. Massera D, McClelland RL, Ambale-Venkatesh B, et al. Prevalence of unexplained left ventricular hypertrophy by cardiac magnetic reso- 
nance imaging in MESA. J Am Heart Assoc 2019; 8: e012250. DOI: 10.1161/JAHA.119.012250.

8. Marian AJ, Braunwald E. Hypertrophic cardiomyopathy. Circ Res 2017; 121: 749-770.

9. Maron BJ. Clinical course and management of hypertrophic cardiomyopathy. N Engl J Med 2018; 379: 655-668.

10. Dorbala S, Ando Y, Bokhari S, et al. ASNC/AHA/ASE/EANM/ HFSA/ISA/SCMR/SNMMI expert consensus recommendations for multimodality imaging in cardiac amyloidosis: part 2 of 2 - diagnostic criteria and appropriate utilization. J Card Fail 2019; 25: 854-865.

11. Prevalence of cardiac amyloidosis among adult patients referred to tertiary centres with an initial diagnosis of hypertrophic cardiomyopathy. Int J Cardiol 2020; 300: 191-195.

12. Kitaoka H, Toru K, Yoshinori LD. Hypertrophic cardiomyopathy a heterogeneous and lifelong disease in the real world. Circ J 2020; 84: $1218-1226$

13. Limongelli G, Masarone D, Verrengia M, et al. Diagnostic clues for the diagnosis of nonsarcomeric hypertrophic cardiomyopathy (phenocopies): amyloidosis, Fabry disease, and mitochondrial disease. J Cardiovasc Echography 2018; 28: 120-123.

14. Fuad JM, Jamil TA. Modern imaging techniques in cardiomyopathies. Circ Res 2017; 121: 874-891.

15. Śpiewak M, Kłopotowski M, Ojrzyńska N, et al. Impact of cardiac magnetic resonance on the diagnosis of hypertrophic cardiomyopathy - a 10-year experience with over 1000 patients. Eur Radiol 2021; 31: 1194-1205

16. Rowin EJ, Maron MS. The role of cardiac MRI in the diagnosis and risk stratification of hypertrophic cardiomyopathy. Arrhythmia Electrophysiol Rev 2016; 5: 197-202.

17. Maron MS, Rowin EJ, Maron BJ. How to image hypertrophic cardiomyopathy. Circ Cardiovasc Imaging 2017; 10: e005372. DOI: 10.1161/ CIRCIMAGING.116.005372.

18. Schnell F, Riding N, O'Hanlon R, et al. Recognition and significance of pathological T-wave inversions in athletes. Circulation 2015; 131: 165-173.

19. Kawel-Boehm N, Hetzel SJ, Ambale-Venkatesh B, et al. Reference ranges ("normal values") for cardiovascular magnetic resonance (CMR) in adults and children: 2020 update. J Cardiovasc Magn Reson 2020; 22: 87.

20. Iles LM, Ellims AH, Llewellyn $\mathrm{H}$, et al. Histological validation of cardiac magnetic resonance analysis of regional and diffuse interstitial myocardial fibrosis. Eur Heart J Cardiovasc Imaging 2015; 16: 14-22.

21. Brenes JC, Doltra A, Prat S. Cardiac magnetic resonance imaging in the evaluation of patients with hypertrophic cardiomyopathy. Glob Cardiol Sci Pract 2018; 2018: 22. DOI: 10.21542/gcsp.2018.22.

22. Baxi AJ, Restrepo CS, Vargas D, et al. Hypertrophic cardiomyopathy from A to Z: genetics, pathophysiology imaging, and management. Radiographics 2016; 36: 335-354.

23. Chan RH, Maron BJ, Olivotto I, et al. Prognostic value of quantitative contrast-enhanced cardiovascular magnetic resonance for the evaluation of sudden death risk in patients with hypertrophic cardiomyopathy. Circulation 2014; 130: 484-495.

24. Weng Z, Yao J, Chan RH, et al. Prognostic value of LGE-CMR in HCM: a meta-analysis. JACC Cardiovasc Imaging 2016; 9: 1392-1402.

25. Maron MS, Rowin EJ, Wessler BS, et al. Enhanced American College of Cardiology/American Heart Association strategy for prevention of sudden cardiac death in high-risk patients with hypertrophic cardiomyopathy. JAMA Cardiol 2019; 4: 644.

26. Avanesov M, Münch J, Weinrich J, et al. Prediction of the estimated 5-year risk of sudden cardiac death and syncope or non-sustained ventricular tachycardia in patients with hypertrophic cardiomyopathy using late gadolinium enhancement and extracellular volume CMR. Eur Radiol 2017; 27: 5136-5145.

27. Thomas D, Luetkens J, Faron A, et al. Feature-tracking-based strain analysis - a comparison of tracking algorithms. Pol J Radiol 2020; 85: 97-103.

28. Klopotowski M, Kukula K, Malek LA, et al. The value of cardiac magnetic resonance and distribution of late gadolinium enhancement for risk stratification of sudden cardiac death in patients with hypertrophic cardiomyopathy. J Cardiol 2016; 68: 49-56.

29. Chacko BR, Karur GR, Connelly KA, et al. Left ventricular structure and diastolic function by cardiac magnetic resonance imaging in hypertrophic cardiomyopathy. Indian Heart J 2018; 70: 75-81.

30. Malek LA, Misko J, Klopotowski M, et al. Left ventricular diastolic function assessed with cardiovascular magnetic resonance imaging and exercise capacity in patients with non-obstructive hypertrophic cardiomyopathy. J Cardiovasc Magn Reson 2009; 11(S1): P238, 1532-429X-11-S1-P238. DOI: 10.1186/1532-429X-11-S1-P238.

31. Webb J, Fovargue L, Tøndel K, et al. The emerging role of cardiac magnetic resonance imaging in the evaluation of patients with HFpEF. Curr Heart Fail Rep 2018; 15: 1-9. DOI: 10.1007/s11897-018-0372-1.

32. Kawaji K, Codella NCF, Prince MR, et al. Automated segmentation of routine clinical cardiac magnetic resonance imaging for assessment of left ventricular diastolic dysfunction. Circ Cardiovasc Imaging 2009; 2: 476-484.

33. Maceira AM, Cosín-Sales J, Roughton M, et al. Reference left atrial dimensions and volumes by steady state free precession cardiovascular magnetic resonance. J Cardiovasc Magn Reson 2010; 12: 65.

34. Maron BJ, Haas TS, Maron MS, et al. Left atrial remodeling in hypertrophic cardiomyopathy and susceptibility markers for atrial fibrillation identified by cardiovascular magnetic resonance. Am J Cardiol 2014; 113: 1394-1400.

35. Farhad H, Seidelmann SB, Vigneault D, et al. Left atrial structure and function in hypertrophic cardiomyopathy sarcomere mutation carriers with and without left ventricular hypertrophy. J Cardiovasc Magn Reson 2017; 19: 107. DOI: 10.1186/s12968-017-0420-0.

36. Semsarian C, Ingles J, Maron MS, Maron BJ. New perspectives on the prevalence of hypertrophic cardiomyopathy. J Am Coll Cardiol 2015; 65: 1249-1254.

37. Maron MS, Olivotto I, Harrigan C, et al. Mitral valve abnormalities identified by cardiovascular magnetic resonance represent a primary phenotypic expression of hypertrophic cardiomyopathy. Circulation 2011; 124: 40-47. DOI: 10.1161/CIRCULATIONAHA.110.985812.

38. Maron MS, Maron BJ, Harrigan C, et al. Hypertrophic cardiomyopathy phenotype revisited after 50 years with cardiovascular magnetic resonance. J Am Coll Cardiol 2009; 54: 220-228.

39. Maron BJ, Maron MS, Maron BA, Loscalzo J. Moving beyond the sarcomere to explain heterogeneity in hypertrophic cardiomyopathy: JACC review topic of the week. J Am Coll Cardiol 2019; 73: 1978-1986.

40. Rowin EJ, Maron BJ, Haas TS, et al. Hypertrophic cardiomyopathy with left ventricular apical aneurysm: implications for risk stratification and management. J Am Coll Cardiol 2017; 69: 761-773. 
41. Fumagalli C, Gregorio MGD, Zampieri M, et al. Targeted medical therapies for hypertrophic cardiomyopathy. Curr Cardiol Rep 2020; 22: 10 .

42. Vogel-Claussen J, Tomas MS, Newatia A, et al. Cardiac MRI evaluation of hypertrophic cardiomyopathy: left ventricular outflow tract/aortic valve diameter ratio predicts severity of LVOT obstruction. J Magn Reson Imaging JMRI 2012; 36: 598-603.

43. Marstrand P, Han L, Day SM, et al. Hypertrophic cardiomyopathy with left ventricular systolic dysfunction. Circulation 2020; 141: 1371-1383.

44. Frustaci A, Verardo R, Caldarulo M, et al. Myocarditis in hypertrophic cardiomyopathy patients presenting acute clinical deterioration. Eur Heart J 2007; 28: 733-740.

45. Cheng S, Choe YH, Ota H, et al. CMR assessment and clinical outcomes of hypertrophic cardiomyopathy with or without ventricular remodeling in the end-stage phase. Int J Cardiovasc Imaging 2018; 34: 597-605

46. Harris KM, Spirito P, Maron MS, et al. Prevalence, clinical profile, and significance of left ventricular remodeling in the end-stage phase of hypertrophic cardiomyopathy. Circulation 2006; 114: 216-225.

47. Coats CJ, Pavlou M, Watkinson OT, et al. Effect of trimetazidine dihydrochloride therapy on exercise capacity in patients with nonobstructive hypertrophic cardiomyopathy: a randomized clinical trial. JAMA Cardiol 2019; 4: 230-235.

48. Wells SB, Maron M, Patel P, et al. End-stage hypertrophic cardiomyopathy revisited: impact of contemporary therapies. J Am Coll Cardiol 2019; 73 (9 Supplement 1): 762.

49. Maron BJ, Rowin EJ, Casey SA, et al. What do patients with hypertrophic cardiomyopathy die from? Am J Cardiol 2016; 117: 434-435. 